\title{
THE EFFECT OF C2 AND C5 SUBSTITUTIONS ON THE NITROGEN LONE PAIR INVERSION IN 1,3-OXAZINES"
}

\author{
J. GUILLERMO CONTRERAS* , S. MARCELA HURTADO
}

\author{
Facultad de Ciencias Quimicas, Universidad de Concepción, casilla 160-C, \\ Concepción, Chile. \\ (Received 1 October 2008 - Accepted 16 January 2008)
}

\begin{abstract}
A series of $\mathrm{N}$-methyl-2- $\mathrm{R}_{1}, \mathrm{R}_{2}-5-\mathrm{R}_{3}, \mathrm{R}_{4}-1,3$-oxazines (where $\mathrm{R}_{1,2}$ are either $\mathrm{H}$ or $\mathrm{p}$-nitro phenyl group and $\mathrm{R}_{3,4}=\mathrm{H}$ or $\mathrm{CH}_{3}$ - group) have been theoretically studied by ab initio methods in the frame of MO theory. Optimized geometries were obtained at HF and B3LYP/6-31G $\mathrm{G}^{* *}$ levels, whereas energies were derived at MP2 and B3LYP levels employing the same basis set. Geometries obtained at DFT level are needed to obtain consistent values and good correlations between the experimental and theoretical ${ }^{1} \mathrm{H}-\mathrm{NMR}$ data. The nitrogen lone pair would be axial orientated in $\mathbf{I}$ and $\mathbf{I I}$, since the N-methyl group lies in the equatorial position in the gas phase, whereas in compounds III and IV the methyl axial conformers increase their concentration, implying that inversion of the nitrogen lone pair takes place in an appreciable extent. PCM method predicts that solvents $\left(\mathrm{CCl}_{4}, \mathrm{CHCl}_{3}, \mathrm{CH}_{2} \mathrm{Cl}_{2}\right)$ produce a significant effect on conformers preference, but just a modest influence on the equilibrium geometries and ${ }^{1} \mathrm{H}-\mathrm{NMR}$ properties.
\end{abstract}

Keywords: 1,3-Oxazines, Nitrogen Inversion, Lone-Pair Inversion Barrier, Substituents Effects

\section{INTRODUCTION}

The study of the spatial requirements of nitrogen nonbonding pairs of electrons imply the determination of the preferred orientation of alkyl group attached to nitrogen in the heterocycle being studied. Using dipole moment measurements, Urbanski ${ }^{1}$ assigned a chair form to N-methyl or ethyl-5-nitro5 -methyl-1,3-oxazines. The analysis of the ${ }^{1} \mathrm{H}$-NMR multiplet pattern and the position of the methylene signals in nitrocyclohexano suggest

that the only species present at room temperature possesses a chair conformation with the nitro group being in an equatorial position ${ }^{2}$. Studying the kinetics of the cycle inversion process in some tetrahydro-1,3-oxazines by variable temperature NMR spectra, Lehn et $\mathrm{al}^{3}$ propose that nitrogen inversion process is faster than the ring inversion so that the ${ }^{1} \mathrm{H}-\mathrm{NMR}$ spectra will show an $\mathrm{AB}$ system for $\mathrm{C} 2$ protons, whereas the $\mathrm{N}-\mathrm{CH}_{3}$ group will show up as single averaged signal. The experimental data support a conformational equilibrium between two equivalent chairs ${ }^{3,4}$. Allingham et $\mathrm{al}^{5}$ extended the Urbinski's series ${ }^{1,6}$ by including the n-propyl, i-propyl and cyclohexyl derivatives. The ${ }^{1} \mathrm{H}-\mathrm{NMR}$ data allow to determine the preferential conformation by studying the $\mathrm{J}_{\mathrm{gem}}$ for $\mathrm{C} 2, \mathrm{C} 4$ and $\mathrm{C} 6$ protons. These coupling constants would be sensitive to orientation of the nitrogen lone pair. Katritzky et $\mathrm{al}^{7}$ found that $\Delta \mathrm{G}^{\circ}{ }_{138}$ is ca. $-0.16 \mathrm{kcal} / \mathrm{mol}$ for the equatorial $\square$ axial equilibrium in 3-methyl-1-oxa3 -azacyclohexane. The mayor component is that having an axial methyl group. The calculated nitrogen inversion barrier is ca. $7.6 \mathrm{kcal} / \mathrm{mol}$, whereas in pyperidine, a value of $8.7 \mathrm{kcal} / \mathrm{mol}^{\text {has }}$ been reported ${ }^{8}$.

In the present work a series of $\mathrm{N}$-methyl-2,5-substituted-1,3-oxazines have been studied with the aim to determine the effect of substitution on C5 and/or $\mathrm{C} 2$ on electronic delocalization, steric factors that might be important in the nitrogen inversion process. On the other hand, it looks promising to study the influence of these substitutions on the NMR properties. In fact, we have paid attention to relations between chemical shifts, ${ }^{1} \mathrm{~J}$ and $\mathrm{J}_{\text {gem }}$ spin-spin coupling constants with energy barriers and hyperconjugative interactions. The inversion barriers for some pairs of species have also been determined and compared with the experimental values when available. Important part of this work is devoted to the search the TSs connecting both conformers in the equatorial $\square$ axial reaction. For this purpose we have assumed that, a nitrogen atom with three substituents and a nonbonding lone pair of electrons has a pyramidal geometry capable of inverting its configuration. The ground state has a nominally a $\mathrm{sp}^{3}$-hybridized nitrogen with the lone pair occupying an orbital that is approximately a $\mathrm{sp}^{3}$ hybrid, and the transition states has an $\mathrm{sp}^{2}$-hybridized nitrogen with the lone pair in a p orbital'. In the present work, the preferred orientation of the methyl group on nitrogen has been studied and compared with the scarce experimental data. The $\mathrm{C} 5$ and $\mathrm{C} 2$ substitution by electron releasing or withdrawing groups allow inferring the effect on the nitrogen inversion barriers. The calculated proton chemical shifts and coupling constants have also been used to derive the relative \% concentration of the axial and equatorial species.

\section{COMPUTATIONAL METHODS} the

Geometry optimizations were carried out at HF and B3LYP levels using

$6-31 \mathrm{G}^{* *}$ basis set. In all the cases, GAUSSIAN 03 series of programs were used ${ }^{10}$. The initial geometries were those previously reported for other oxazines ${ }^{11}$. Harmonic vibrational frequencies and IR intensities were calculated at the equilibrium geometries. In all cases, the equilibrium geometries yielded all real frequencies and thereby the 8 derived structures at either HF or B3LYP/6-31G** levels are local minima. Energy calculations were carried out using various basis sets in order to obtain the optimum level to be used in the calculation of the NMR properties. GIAO (Gauge-Independent Atomic Orbital) method ${ }^{12,13}$ was applied to derive the magnetic tensors. Chemical shits (in ppm) were referred to tetramethylsilane (TMS) standard (isotropic magnetic tensor $=32.1 \mathrm{ppm}$ for $\left.{ }^{1} \mathrm{H}\right)^{14}$. Natural Bond Orbital ${ }^{15,16}$ analysis was performed to study the most prominent interactions in each molecular structure. NBO analysis allows the separation of the molecular energy into two fundamental contributions: the total energy (where delocalization is included), and the energy of the hypothetical Lewis structure. Accordingly, the electron are strictly located in bonds and lone pairs. The interactions between filled and antibonding (or Rydberg) orbitals represent the deviation of the molecule from the ideal Lewis structure and can be used as a measure of delocalizations $\left(\mathrm{E}_{\text {deloc }}\right.$ $=E_{\text {at }}-E_{\text {towi }}$. The energy of the hypothetical Lewis structure with strictly localized bonds is obtained by removing all off-diagonal elements from the Fock matrix and computing one SCF cycle. The hyperconjugation interaction energies were obtained from the second-order perturbation approach:

$\mathrm{E}_{(2)}=-2<\sigma \nabla \mathrm{F} \otimes \sigma \bigotimes>^{2} / \varepsilon_{\mathrm{\sigma} \bigotimes}-\varepsilon_{\sigma}$, where the integral corresponds to the $\mathrm{F}_{\mathrm{ij}}$ matrix element between interacting orbitals $i$ and $j$ whose energies are $\varepsilon_{\sigma}$ and $\varepsilon_{\sigma 区} .2$ stands for the population of the donor orbital. There is good linear correlation between the second-order perturbation hyperconjugative energies and the deletion procedure ${ }^{17}$. All the NBO calculations were carried out at B3LYP/6-31G ${ }^{* *}$. To obtain the theoretical ${ }^{1} \mathrm{H}$ and ${ }^{13} \mathrm{C}$ chemical shifts $(\delta)$, the magnetic shielding tensors were calculated at B3LYP/ 6-311+ $\mathrm{G}^{* *} / / \mathrm{B} 3 \mathrm{LYP} / 6-$ $31 \mathrm{G}^{* *}$ level of theory ${ }^{14}$ using the Gauge- Independent Atomic Orbital (GIAO) method ${ }^{15}$. Chemical shifts were derived regarding to the NMR isotropic magnetic shielding tensors (in ppm) from the corresponding standard tensors (TMS, $31.904 \mathrm{ppm}$ for ${ }^{1} \mathrm{H}$ and $183.436 \mathrm{ppm}$ for ${ }^{13} \mathrm{C}$ ).

Since TS are local maxima in the reaction pathways, they are no accessible to experimental studies and thereby no direct information on their structures are available. Theoretical methods are the only way to infer some knowledge about transition status at present. Hammond has suggested ${ }^{20}$ that the energetic barrier is closely related with the reactants and products structures. Thus, Hammond's postulate is a useful tool to connect the energy barrier position in the potential energy surface (PES) with the exo or endothermic character of the reaction. Accordingly, if the TS is close in energy to a stable species, its structure will be alike. Brönsted coefficient (eq.1) and Marcus equation (eq.2) allow to locate a TS. 


$$
\beta=\frac{1}{2}+\frac{\Delta E^{0}}{8 \Delta E_{0}^{+}}
$$

$$
\Delta E^{*}=\Delta E_{0}^{*}+\frac{1}{2} \Delta E^{0}+\frac{\left(\Delta E^{0}\right)^{2}}{16 \Delta E_{0}^{*}}
$$

where: $\Delta \mathrm{E}^{\ddagger}$ is the activation energy, and $\Delta \mathrm{E}^{0}$ corresponds to the reaction energy. According to these equations an exothermic reaction will possess a TS similar to the reactant and $\beta$ will be $<1 / 2$.. When $\beta$ is $>1 / 2$ the TS resembles the products, and the reaction will be an endothermic one..

To model the solvent-solute interaction the polarizable continuum model $(\mathrm{PCM})^{21}$, was employed. PCM considers the solvent as a continued unstructured medium whose polarization can be represented by a macroscopic constant such as the dielectric constant, $\varepsilon$. The cavity hosting the solute is constructed with a number of overlapping spheres centered at the solute nuclei and possessing radii proportional to the Van Der Waals ones. The solute induces a polarization of the medium that brings on a charge density in the cavity surface in turn. Our large experience in our laboratory using this method to derived solvation free energies, allows us to be confident in the solvent modelation.

\section{RESULTS AND DISCUSSION}

\section{MOLECULAR STRUCTURES}

1,3-oxazine is a saturated six-member heterocycle containing both nitrogen and oxygen with a distorted chair-like structure. Oxazines derivatives studied here are distinguished by their substituents as well as the orientation of the $\mathrm{CH}_{3}$ group. The designation axial or equatorial refers to the orientation of the methyl group attached to $\mathrm{N}$ atom, whereas I, II , III and IV refers to the type of substituents on $\mathrm{C} 2$ and $\mathrm{C} 5$ atoms (see figure 1).

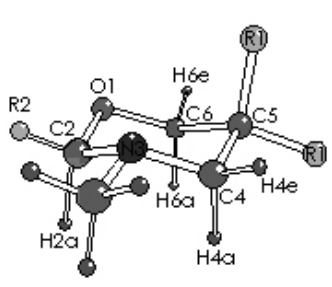

equatorial form

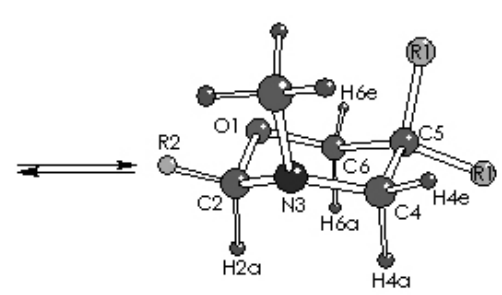

axial form
I) $\mathrm{R} 1=\mathrm{CH}_{3} \quad \mathrm{R} 2=\mathrm{H}$

II) $\mathrm{R} 1=\mathrm{CH}_{3} \quad \mathrm{R} 2=\mathrm{p}-\mathrm{NO} 2 \cdot \mathrm{C} 6 \mathrm{H} 4$

III) $\mathrm{R} 1=\mathrm{H}^{3} \quad \mathrm{R} 2=\mathrm{H}$

V) $\mathrm{R} 1=\mathrm{H} \quad \mathrm{R} 2=\mathrm{p}-\mathrm{NO} 2 \cdot \mathrm{C} 6 \mathrm{H} 4$

Figure 1.- Equatorial and Axial conformers of N-methyl-1,3-oxazine derivatives. The atom numbering is used throughout the paper

From table 1, it can be inferred that difference between the dihedral angles that define each side of the ring, $\mathrm{C} 2 \mathrm{O} 1 \mathrm{C} 6 \mathrm{C} 5$ and $\mathrm{C} 2 \mathrm{~N} 3 \mathrm{C} 4 \mathrm{C} 5(\Delta \tau)$, show that Iax and IIax conformers are more distorted at the $\mathrm{O}$ than at the $\mathrm{N}$-side. In fact, the presence of two methyl groups on C5 leads $\Delta \tau$ values of ca. 11-14 , whereas in the corresponding equatorial conformers $\Delta \tau$ are ca $3-4^{\circ}$ only. The presence of a large substituent on $\mathrm{C} 2$ seems to exert no much influence on the geometry of the oxazine ring since $\Delta \tau$ is just ca. $4^{\circ}$ for both axial and equatorial IV derivatives. IIIax is an exception as no substituents on $\mathrm{C} 2$ or $\mathrm{C} 5$ are present and behaves like I and II axial conformers, though to a lesser extend.
Important $\mathrm{C}-\mathrm{H}$ bond distances are $\mathrm{C} 2-\mathrm{H} 2 \mathrm{a}$ and $\mathrm{C} 4-\mathrm{H} 4 \mathrm{a}$ for being the ones varying more within this series of conformers. These distances are longer in the equatorial conformers, implying a delocalization of the type (LP)N3 $\rightarrow \sigma^{*}$ $\mathrm{C} 2-\mathrm{H} 2 \mathrm{a}$ and $(\mathrm{LP}) \mathrm{N} 3 \rightarrow \sigma^{*} \mathrm{C} 4-\mathrm{H} 4 \mathrm{a}$ that contribute to the molecular stability. Larger $\mathrm{C}-\mathrm{H}$ bond lengths dependence with $\mathrm{C} 2$ and $\mathrm{C} 5$ substitutions is observed in the equatorial conformers. Shorter $\mathrm{C} 6 \mathrm{Ha}$ distance occurs in all cases but IIIeq. The plot of ring relative (with respect conformer III) bond distances vs. the corresponding distances for all other conformers studied here, allow to remove any other effect, such as lone-pairs delocalization or other related to the substituent position but the one produced by the methyl groups on $\mathrm{C} 5$ (or $\mathrm{C} 2$ for the p-nitrophenyl group). Fig.2 shows that the increase of the C4C5 AND C5 6 bond lengths are due to the presence of two $\mathrm{CH}_{3}$ - groups in conformers I and II. The p-nitrophenyl group in conformers II and IV lead to larger C2N3 distances. Certain degree of linearity in the $\mathrm{C} 2 \mathrm{~N} 3$ distances throughout this series of conformers: I $<$ III $<$ II $<$ IV.

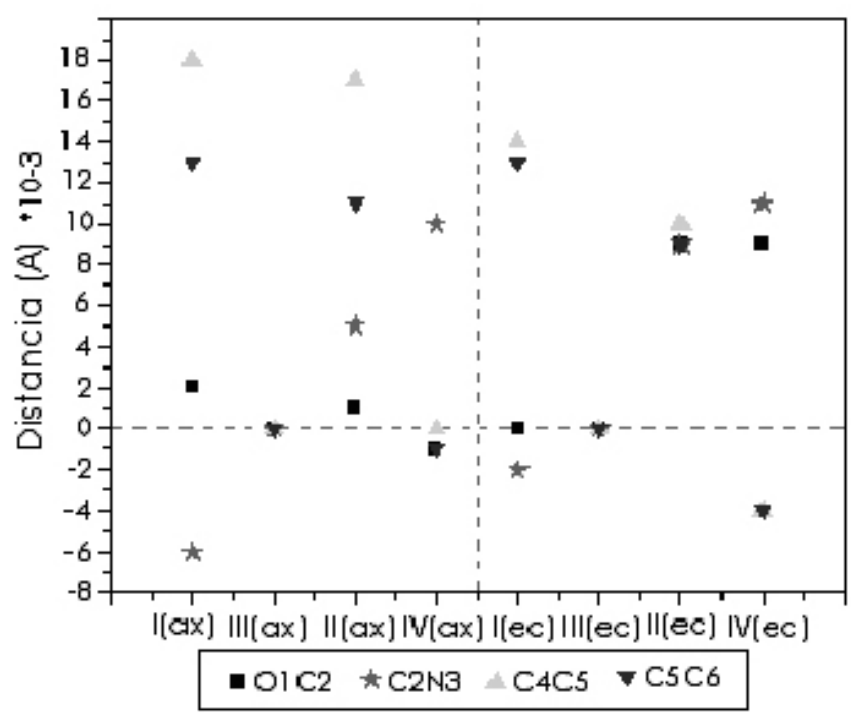

Figura 2.- Relative distances with respect to conformer III.

Since p-nitrophenyl is a strong electron-withdrawing group, weakens and enlarges the $\mathrm{C} 2 \mathrm{~N} 3$ distance, whereas methyl being an electron-releasing group counterbalances the above effect in conformer II. The $\mathrm{O} 1 \mathrm{C} 2$ bond distance found for the series of conformers of these substituted oxazines are longer for II and IV most probably due to the p-nitrophenyl group presence. The substituent on N3 in an equatorial position facilitates the chain/ring tautomerization in 1,3 -oxazines ${ }^{18}$. The chain/ring tautomerism have been extensively studied by means IR a UV spectroscopies ${ }^{19}$ and shown that the tautomeric equilibrium takes place due to the substituent located in the aromatic ring. Thus, electronwithdrawing groups increase the cyclic form contribution, whereas electronreleasing substituents would favor the chain forms. In the axial structures, the O1C2N3 AND C5C4N3 angles are larger than the corresponding angles in the equatorial conformers. These angles are related to the anomeric effect so essential for the axial conformations. Considering these angles in structure IIIax and taking into account that 1,3-diaxial repulsions between the $\mathrm{N}$-substituent and $\mathrm{Ha}$ on $\mathrm{C} 5$ that are very small, the methyl(ax) group effect on $\mathrm{C} 5$ can be determined, as well as the effect on angle O1C2N3 exerted by the p-nitrophenyl group. The variation of the above mentioned angles for al species studied here are shown in table 2 . 
Table 1.- B3LYP/6-31G** selected structural parameters for the substituted 1,3-oxazinas

\begin{tabular}{|c|c|c|c|c|c|c|c|c|}
\hline \multirow{2}{*}{ Parameter ${ }^{\mathrm{a}, \mathrm{b}}$} & \multicolumn{2}{|c|}{ I } & \multicolumn{2}{|c|}{ II } & \multicolumn{2}{|c|}{ III } & \multicolumn{2}{|c|}{ IV } \\
\hline & Axial & Equatorial & Axial & Equatorial & Axial & Equatorial & Axial & Ecuatorial \\
\hline $\mathrm{O} 1-\mathrm{C} 2$ & $\begin{array}{l}1.433 \\
(1.403)\end{array}$ & $\begin{array}{c}1.408 \\
(1.386)\end{array}$ & $\begin{array}{c}1.432 \\
(1.401)\end{array}$ & $\begin{array}{c}1.417 \\
(1.392)\end{array}$ & $\begin{array}{c}1.431 \\
(1.403)\end{array}$ & $\begin{array}{c}1.408 \\
(1.387)\end{array}$ & $\begin{array}{c}1.430 \\
(1.400)\end{array}$ & $\begin{array}{c}1.417 \\
(1.392)\end{array}$ \\
\hline $\mathrm{C} 2-\mathrm{N} 3$ & $\begin{array}{c}1.438 \\
(1.430)\end{array}$ & $\begin{array}{l}1.456 \\
(1.443)\end{array}$ & $\begin{array}{c}1.449 \\
(1.438)\end{array}$ & $\begin{array}{c}1.467 \\
(1.451)\end{array}$ & $\begin{array}{c}1.444 \\
(1.433)\end{array}$ & $\begin{array}{l}1.458 \\
(1.444)\end{array}$ & $\begin{array}{c}1.454 \\
(1.442)\end{array}$ & $\begin{array}{l}1.469 \\
(1.453)\end{array}$ \\
\hline N3-C4 & $\begin{array}{c}1.459 \\
(1.456)\end{array}$ & $\begin{array}{c}1.463 \\
(1.451)\end{array}$ & $\begin{array}{l}1.470 \\
(1.459)\end{array}$ & $\begin{array}{c}1.468 \\
(1.457)\end{array}$ & $\begin{array}{c}1.471 \\
(1.458)\end{array}$ & $\begin{array}{c}1.463 \\
(1.451)\end{array}$ & $\begin{array}{c}1.474 \\
(1.461)\end{array}$ & $\begin{array}{l}1.468 \\
(1.457)\end{array}$ \\
\hline $\mathrm{C} 4-\mathrm{C} 5$ & $\begin{array}{c}1.558 \\
(1.549)\end{array}$ & $\begin{array}{l}1.546 \\
(1.538)\end{array}$ & $\begin{array}{c}1.557 \\
(1.548)\end{array}$ & $\begin{array}{c}1.542 \\
(1.534)\end{array}$ & $\begin{array}{l}1.540 \\
(1.534)\end{array}$ & $\begin{array}{c}1.532 \\
(1.526)\end{array}$ & $\begin{array}{l}1.540 \\
(1.533)\end{array}$ & $\begin{array}{l}1.528 \\
(1.522)\end{array}$ \\
\hline $\mathrm{C} 5-\mathrm{C} 6$ & $\begin{array}{c}1.546 \\
(1.536)\end{array}$ & $\begin{array}{c}1.541 \\
(1.532)\end{array}$ & $\begin{array}{c}1.544 \\
(1.536)\end{array}$ & $\begin{array}{c}1.537 \\
(1.528)\end{array}$ & $\begin{array}{c}1.533 \\
(1.526)\end{array}$ & $\begin{array}{c}1.528 \\
(1.522)\end{array}$ & $\begin{array}{l}1.532 \\
(1.526)\end{array}$ & $\begin{array}{c}1.524 \\
(1.517)\end{array}$ \\
\hline N3-R & $\begin{array}{l}1.459 \\
(1.450)\end{array}$ & $\begin{array}{l}1.454 \\
(1.444)\end{array}$ & $\begin{array}{l}1.465 \\
(1.456)\end{array}$ & $\begin{array}{l}1.461 \\
(1.451)\end{array}$ & $\begin{array}{l}1.462 \\
(1.452)\end{array}$ & $\begin{array}{l}1.453 \\
(1.444)\end{array}$ & $\begin{array}{l}1.468 \\
(1.457)\end{array}$ & $\begin{array}{l}1.461 \\
(1.451)\end{array}$ \\
\hline $\mathrm{C} 2-\mathrm{H} 2 \mathrm{a}$ & $\begin{array}{c}1.103 \\
(1.090)\end{array}$ & $\begin{array}{l}1.108 \\
(1.100)\end{array}$ & $\begin{array}{c}1.105 \\
(1.091)\end{array}$ & $\begin{array}{c}1.114 \\
(1.098)\end{array}$ & $\begin{array}{c}1.103 \\
(1.089)\end{array}$ & $\begin{array}{l}1.116 \\
(1.100)\end{array}$ & $\begin{array}{c}1.105 \\
(1.090)\end{array}$ & $\begin{array}{l}1.114 \\
(1.098)\end{array}$ \\
\hline $\mathrm{C} 2-\mathrm{H} 2 \mathrm{e}$ & $\begin{array}{c}1.092 \\
(1.081)\end{array}$ & $\begin{array}{c}1.095 \\
(1.082)\end{array}$ & ------- & -------- & $\begin{array}{c}1.093 \\
(1.081)\end{array}$ & $\begin{array}{c}1.094 \\
(1.082)\end{array}$ & -------- & ------- \\
\hline $\mathrm{C} 4-\mathrm{H} 4 \mathrm{a}$ & $\begin{array}{c}1.100 \\
(1.089)\end{array}$ & $\begin{array}{c}1.111 \\
(1.098)\end{array}$ & $\begin{array}{c}1.100 \\
(1.088)\end{array}$ & $\begin{array}{l}1.110 \\
(1.097)\end{array}$ & $\begin{array}{c}1.099 \\
(1.088)\end{array}$ & $\begin{array}{c}1.111 \\
(1.098)\end{array}$ & $\begin{array}{c}1.098 \\
(1.088)\end{array}$ & $\begin{array}{l}1.110 \\
(1.097)\end{array}$ \\
\hline $\mathrm{C} 4-\mathrm{H} 4 \mathrm{e}$ & $\begin{array}{c}1.096 \\
(1.085)\end{array}$ & $\begin{array}{c}1.097 \\
(1.086)\end{array}$ & $\begin{array}{c}1.095 \\
(1.084)\end{array}$ & $\begin{array}{c}1.096 \\
(1.085)\end{array}$ & $\begin{array}{c}1.095 \\
(1.084)\end{array}$ & $\begin{array}{c}1.096 \\
(1.085)\end{array}$ & $\begin{array}{c}1.094 \\
(1.084)\end{array}$ & $\begin{array}{c}1.095 \\
(1.084)\end{array}$ \\
\hline C6-H6a & $\begin{array}{c}1.105 \\
(1.092)\end{array}$ & $\begin{array}{c}1.104 \\
(1.092)\end{array}$ & $\begin{array}{c}1.104 \\
(1.091)\end{array}$ & $\begin{array}{c}1.103 \\
(1.091)\end{array}$ & $\begin{array}{c}1.105 \\
(1.092)\end{array}$ & $\begin{array}{c}1.111 \\
(1.092)\end{array}$ & $\begin{array}{c}1.104 \\
(1.091)\end{array}$ & $\begin{array}{c}1.103 \\
(1.091)\end{array}$ \\
\hline C6-H6e & $\begin{array}{c}1.095 \\
(1.084)\end{array}$ & $\begin{array}{c}1.095 \\
(1.083)\end{array}$ & $\begin{array}{c}1.095 \\
(1.083)\end{array}$ & $\begin{array}{c}1.094 \\
(1.083)\end{array}$ & $\begin{array}{c}1.094 \\
(1.083)\end{array}$ & $\begin{array}{c}1.094 \\
(1.082)\end{array}$ & $\begin{array}{c}1.093 \\
(1.082)\end{array}$ & $\begin{array}{l}1.093 \\
(1.082)\end{array}$ \\
\hline$<\mathrm{O} 1 \mathrm{C} 2 \mathrm{~N} 3$ & $\begin{array}{c}114.3 \\
(113.4)\end{array}$ & $\begin{array}{c}111.7 \\
(111.4)\end{array}$ & $\begin{array}{l}113.0 \\
(112.2)\end{array}$ & $\begin{array}{c}110.3 \\
(110.3)\end{array}$ & $\begin{array}{c}114.4 \\
(113.6)\end{array}$ & $\begin{array}{l}112.1 \\
(111.7)\end{array}$ & $\begin{array}{l}113.2 \\
(112.6)\end{array}$ & $\begin{array}{l}110.8 \\
(110.7)\end{array}$ \\
\hline$<\mathrm{C} 5 \mathrm{C} 4 \mathrm{~N} 3$ & $\begin{array}{c}115.6 \\
(115.4)\end{array}$ & $\begin{array}{c}111.0 \\
(110.9)\end{array}$ & $\begin{array}{c}115.9 \\
(115.5)\end{array}$ & $\begin{array}{c}112.4 \\
(112.2)\end{array}$ & $\begin{array}{c}112.6 \\
(112.4)\end{array}$ & $\begin{array}{c}109.6 \\
(109.5)\end{array}$ & $\begin{array}{c}112.5 \\
(112.2)\end{array}$ & $\begin{array}{c}110.7 \\
(110.7)\end{array}$ \\
\hline$<\mathrm{H} 2 \mathrm{aC} 2 \mathrm{H} 2 \mathrm{e}$ & $\begin{array}{c}109.3 \\
(108.8)\end{array}$ & $\begin{array}{c}108.5 \\
(108.4)\end{array}$ & ------ & --------- & $\begin{array}{c}109.3 \\
(108.9)\end{array}$ & $\begin{array}{c}108.4 \\
(108.4)\end{array}$ & -------- & -------- \\
\hline$<\mathrm{H} 4 \mathrm{aC} 4 \mathrm{H} 4 \mathrm{e}$ & $\begin{array}{c}106.8 \\
(106.7)\end{array}$ & $\begin{array}{c}107.0 \\
(107.0)\end{array}$ & $\begin{array}{c}106.9 \\
(106.6)\end{array}$ & $\begin{array}{c}106.8 \\
(106.9)\end{array}$ & $\begin{array}{c}107.0 \\
(106.9)\end{array}$ & $\begin{array}{c}107.0 \\
(107.1)\end{array}$ & $\begin{array}{c}107.1 \\
(106.9)\end{array}$ & $\begin{array}{c}106.9 \\
(107.0)\end{array}$ \\
\hline$<\mathrm{H6aC6H6e}$ & $\begin{array}{c}107.8 \\
(107.8)\end{array}$ & $\begin{array}{c}108.1 \\
(108.0)\end{array}$ & $\begin{array}{c}107.9 \\
(107.8)\end{array}$ & $\begin{array}{c}108.2 \\
(108.0)\end{array}$ & $\begin{array}{c}107.8 \\
(107.8)\end{array}$ & $\begin{array}{c}108.1 \\
(108.0)\end{array}$ & $\begin{array}{c}107.9 \\
(107.9)\end{array}$ & $\begin{array}{c}108.1 \\
(108.1)\end{array}$ \\
\hline$<\mathrm{C} 4 \mathrm{C} 5 \mathrm{C} 6$ & $\begin{array}{c}106.8 \\
(107.1)\end{array}$ & $\begin{array}{c}106.6 \\
(106.8)\end{array}$ & $\begin{array}{c}106.8 \\
(107.3)\end{array}$ & $\begin{array}{c}106.2 \\
(106.4)\end{array}$ & $\begin{array}{c}109.1 \\
(109.2)\end{array}$ & $\begin{array}{c}109.4 \\
(109.4)\end{array}$ & $\begin{array}{c}109.3 \\
(109.4)\end{array}$ & $\begin{array}{c}108.8 \\
(108.7)\end{array}$ \\
\hline$<\mathrm{C} 2 \mathrm{O} 1 \mathrm{C} 6 \mathrm{C} 5\left(\tau_{1}\right)$ & $\begin{array}{c}61.3 \\
(61.0)\end{array}$ & $\begin{array}{c}59.5 \\
(59.1)\end{array}$ & $\begin{array}{c}60.8 \\
(60.3)\end{array}$ & $\begin{array}{c}60.6 \\
(60.1)\end{array}$ & $\begin{array}{r}57.9 \\
(57.6)\end{array}$ & $\begin{array}{r}58.0 \\
(57.6)\end{array}$ & $\begin{array}{r}57.4 \\
(57.0)\end{array}$ & $\begin{array}{r}59.2 \\
(58.9)\end{array}$ \\
\hline$<\mathrm{C} 2 \mathrm{~N} 3 \mathrm{C} 4 \mathrm{C} 5\left(\tau_{2}\right)$ & $\begin{array}{c}47.8 \\
(47.5)\end{array}$ & $\begin{array}{c}56.7 \\
(55.9)\end{array}$ & $\begin{array}{c}50.1 \\
(50.2)\end{array}$ & $\begin{array}{c}56.2 \\
(55.4)\end{array}$ & $\begin{array}{c}51.7 \\
(51.8)\end{array}$ & $\begin{array}{c}55.8 \\
(55.2)\end{array}$ & $\begin{array}{c}53.7 \\
(54.0)\end{array}$ & $\begin{array}{c}55.4 \\
(54.6)\end{array}$ \\
\hline
\end{tabular}

a) Bond distances and angles in $\AA$ and degrees, respectively. b) HF/6-31G** values are given in parentheses.

Table 2. Relative values of $\mathrm{O} 1 \mathrm{C} 2 \mathrm{~N} 3$ and $\mathrm{C} 5 \mathrm{C} 4 \mathrm{~N} 3$ angles referred to conformer III

\begin{tabular}{|c|c|c|c|c|c|c|c|c|}
\hline & $\begin{array}{c}\text { I } \\
(\mathrm{ax})\end{array}$ & $\begin{array}{c}\text { II } \\
(\mathrm{ax})\end{array}$ & $\begin{array}{c}\text { III } \\
(\mathrm{ax})\end{array}$ & $\begin{array}{c}\text { IV } \\
(\mathrm{ax})\end{array}$ & $\begin{array}{c}\text { I } \\
(\mathrm{eq})\end{array}$ & $\begin{array}{c}\text { II } \\
(\mathrm{eq})\end{array}$ & $\begin{array}{c}\text { III } \\
(\mathrm{eq})\end{array}$ & $\begin{array}{c}\text { IV } \\
(\mathrm{eq})\end{array}$ \\
\hline$<\mathrm{O} 1 \mathrm{C} 2 \mathrm{~N} 3$ & -0.1 & -1.4 & 0 & -1.2 & -0.4 & -1.8 & 0 & -1.3 \\
\hline$<\mathrm{C} 5 \mathrm{C} 4 \mathrm{~N} 3$ & 3 & 3.3 & 0 & -0.1 & 1.4 & 2.8 & 0 & 1.1 \\
\hline
\end{tabular}

The value shown by angle $\mathrm{C} 5 \mathrm{C} 4 \mathrm{~N} 3$ reflects the contribution of the anomeric effect and the 1,3-diaxial repulsive interactions producing an increase of ca. 2 and 3 degrees in the equatorial and axial structures, respectively. Angle
O1C2N3 shows a rather modest influence of the concomitant effects already mentioned. The increase in this angle is due to the anomeric effect whereas the p-nitrophenyl decreases such angles to ca 1.5 degrees.

The ${ }^{1} \mathrm{H}-\mathrm{NMR}$ parameters for all conformers were calculated applying the GIAO method and compared with the experimental values previously reported by Lenh et al. ${ }^{3}$ Good agreement of calculated and experimental values support well or model for the local minima found for all conformers. The results are given in table 3 
Table 3. ${ }^{1} \mathrm{H}-\mathrm{NMR}$ chemical shifts (in ppm) and some coupling constants (in Hz) for the N-Methyl-5-substituted- 1,3-oxazines

\begin{tabular}{|c|c|c|c|c|c|c|c|c|c|c|c|c|c|c|c|c|c|c|c|c|c|c|}
\hline \multirow{3}{*}{ Núcleo } & \multicolumn{5}{|c|}{ I } & \multicolumn{5}{|c|}{ II } & \multicolumn{7}{|c|}{ III } & \multicolumn{5}{|c|}{ IV } \\
\hline & Axial & & $\begin{array}{l}\text { Equa- } \\
\text { torial }\end{array}$ & & $\operatorname{Exp}^{c}$ & Axial & & $\begin{array}{l}\text { Equa- } \\
\text { torial }\end{array}$ & & $\operatorname{Exp}^{d}$ & Axial & & $\begin{array}{l}\text { Equa- } \\
\text { torial }\end{array}$ & & Exp & & & Axial & & $\begin{array}{l}\text { Equa- } \\
\text { torial }\end{array}$ & & $\operatorname{Exp}^{d}$ \\
\hline & Gas & Soln $^{c}$ & Gas & Soln ${ }^{c}$ & & Gas & Soln $^{d}$ & Gas & Soln $^{d}$ & & Gas & $\operatorname{Soln}^{c}$ & Gas & Soln & & $\mathrm{C}_{6} \mathrm{H}_{6}$ & $\mathrm{CFCl}_{3}$ & Gas & Soln ${ }^{d}$ & Gas & Soln $^{d}$ & \\
\hline $\mathrm{H}_{2} \mathrm{a}^{\mathrm{a}}$ & 4.38 & 4.44 & 3.34 & 3.40 & 3.27 & 5.39 & 5.47 & 4.12 & 4.26 & 4.21 & 4.54 & 4.60 & 3.46 & 3.53 & & 4.10 & 3.86 & 5.58 & 5.66 & 4.26 & 4.39 & 4.82 \\
\hline $\mathrm{H} 2 \mathrm{e}^{\mathrm{a}}$ & 4.42 & 4.42 & 4.38 & 4.37 & 4.39 & ----- & ----- & ----- & ----- & & 4.36 & 4.36 & 4.35 & 4.34 & & 4.10 & 4.18 & ----- & ----- & ----- & ----- & \\
\hline $\mathrm{H} \mathrm{a}^{\mathrm{a}}$ & 3.27 & 3.35 & 3.04 & 3.12 & 3.08 & 3.62 & 3.69 & 3.23 & 3.32 & & 3.68 & 3.77 & 3.30 & 3.39 & & 3.55 & 3.43 & 4.03 & 4.10 & 3.52 & 3.60 & \\
\hline $\mathrm{H}^{\mathrm{a}} \mathrm{e}^{\mathrm{a}}$ & 3.61 & 3.61 & 3.51 & 3.51 & 3.49 & 3.93 & 3.94 & 3.62 & 3.62 & & 4.05 & 4.05 & 3.95 & 3.95 & & 3.55 & 3.95 & 4.34 & 4.36 & 4.06 & 4.07 & \\
\hline $\mathrm{H}_{4} \mathrm{a}^{\mathrm{a}}$ & 2.92 & 2.96 & 1.72 & 1.79 & 1.72 & 3.26 & 3.30 & 1.99 & 2.07 & 2.15 & 3.18 & 3.23 & 2.00 & 2.08 & & 2.25 & 2.60 & 3.42 & 3.48 & 2.30 & 2.38 & \\
\hline $\mathrm{H} 4 \mathrm{e}^{\mathrm{a}}$ & 2.47 & 2.54 & 2.40 & 2.46 & 2.53 & 2.77 & 2.82 & 2.60 & 2.64 & 2.30 & 2.81 & 2.86 & 2.82 & 2.87 & & 2.25 & 2.83 & 3.09 & 3.13 & 2.99 & 3.02 & \\
\hline $\mathrm{NCH}_{3}{ }^{\mathrm{a}}$ & & & & & & & 2.28 & & 1.78 & 1.90 & & & & & & & & & 1.71 & & 1.40 & 2.05 \\
\hline $\mathrm{J} 2 \mathrm{e}_{\mathrm{e}} \mathrm{a}_{\mathrm{a}}^{\mathrm{b}}$ & 11.91 & & -8.60 & & 7.5 & ----- & & ---- & & ----- & 11.76 & & -8.81 & & & & 9.5 & ----- & & ----- & & \\
\hline $\mathrm{J} 6_{\mathrm{e}} \mathrm{C}_{\mathrm{a}}^{\mathrm{b}}$ & 12.07 & & 11.95 & & 10.8 & 12.25 & & 12.06 & & 11.5 & 12.33 & & 12.22 & & & & 11.5 & 12.50 & & 12.32 & & \\
\hline $\mathrm{J} 4 \mathrm{c}_{\mathrm{a}} \mathrm{b}^{\mathrm{b}}$ & 15.22 & & 11.92 & & 11.0 & 15.20 & & 12.49 & & 11.5 & 15.01 & & 12.14 & & & & & 14.97 & & 12.62 & & \\
\hline $\mathrm{J} 4 \mathrm{e}_{\mathrm{e}} 6_{\mathrm{e}}^{\mathrm{b}}$ & 1.81 & & 1.50 & & 2.2 & 1.84 & & 1.57 & & 2.3 & 1.37 & & 0.81 & & & & & 1.36 & & 0.93 & & \\
\hline $\mathrm{J} 2 \mathrm{e}_{\mathrm{e}} \mathrm{e}_{\mathrm{b}}^{\mathrm{b}}$ & 1.22 & & 0.66 & & 1.3 & ----- & & ----- & & & 1.19 & & 0.71 & & & & & ---- & & ---- & & \\
\hline
\end{tabular}

a)Calculated at B3LYP/6-311+G**// B3LYP/6-31G**. (b) Calculated at B3LYP/6-31G**// B3LYP/6-31G**. c)in: $\mathrm{CH} 2 \mathrm{Cl} 2$; (d)in $\mathrm{CHCl}$

The theoretical NMR chemical shifts $(\delta)$ were calculated at the B3LYP/6$311+\mathrm{G}^{* *} / / \mathrm{B} 3 \mathrm{LYP} / 6-31 \mathrm{G}^{* *}$ level, whereas the coupling constants $(\mathrm{J})$ were derived at $\mathrm{B} 3 \mathrm{LYP} / 6-31 \mathrm{G}^{* *} / / \mathrm{B} 3 \mathrm{LYP} / 6-31 \mathrm{G}^{* *}$ in $\mathrm{CH}_{2} \mathrm{Cl}_{2}$ or $\mathrm{CHCl}_{3}$ solutions. Experimental values of $\delta$ and $\mathrm{J}$ were found to correlate well with the theoretical results obtained at the B3LYP/6-311+G**//B3LYP/6-31G* level of theory. Table 3 shows this agreement for the $\delta$ and $\mathrm{J}$ values. ${ }^{1} \mathrm{H}-\mathrm{NMR}$ spectra ${ }^{4,7}$ in $\mathrm{CF}_{2} \mathrm{Cl}_{2}(\varepsilon=3.50)$ suggest that the conformational equilibrium IIIa $\otimes$ IIIeq is partially displaced to the equatorial form. In fact, Lehn et $\mathrm{al}^{4}$ reported that at the equilibrium, a $45 \%$ of the axial conformer is present, whereas the equatorial is in a $55 \%$ concentration. For the IV species, a $57 \%$ of equatorial and a $43 \%$ of the axial conformer was found. Analysis of the equilibrium between the two forms of III by Katrizky indicates that $\Delta \mathrm{G}^{\mathrm{o}}{ }_{138}=-0.16 \mathrm{kcal} / \mathrm{mol}$ implying a $56.72 \%$ of the axial conformer, i.e. reversing the results of Lehn et al. The strong 1,3 interactions in axial conformers I and II, allow to expect, confidently, that equatorial form will be the predominant one at the equilibrium. Comparing the average value between $\mathrm{CCl}_{4}$ and $\mathrm{CHCl}_{3}$, for compound III a value of ca. $51.11 \%$ of the axial component, can be predicted. This is a value halfway of the reported by Lehn and Katrizky. Closer values to Lehn can be obtained by employing solvents with larger dielectric constants. Axial conformer can achieve concentration of ca. $60 \%$ in compound IV. Table 4 shows relative concentration $\%$ of the axial component in various solvents.

Table 4.- Free energy change in solution $(\mathrm{kcal} / \mathrm{mol})$ and relative concentration of axial components ${ }^{\mathrm{a}}$ in various solvents

\begin{tabular}{|c|l|c|c|}
\hline Compound & Solvent & $\Delta \mathrm{G}_{\text {solution }}^{\circ}$ & Axial\% \\
\hline \multirow{4}{*}{ I } & $\mathrm{CCl}_{4}$ & 1.70 & 5.31 \\
\cline { 2 - 4 } & $\mathrm{CHCl}_{3}$ & 1.74 & 4.99 \\
\cline { 2 - 4 } & $\mathrm{CH}_{2} \mathrm{Cl}_{2}$ & 1.75 & 4.91 \\
\hline \multirow{3}{*}{ II } & $\mathrm{CCl}_{4}$ & 1.40 & 8.64 \\
\cline { 2 - 4 } & $\mathrm{CHCl}_{3}$ & 1.53 & 7.06 \\
\cline { 2 - 4 } & $\mathrm{CH}_{2} \mathrm{Cl}_{2}$ & 1.57 & 6.62 \\
\hline \multirow{6}{*}{$\mathrm{III}$} & $\mathrm{CCl}_{4}$ & -0.07 & 52.80 \\
\cline { 2 - 4 } & $\mathrm{CHCl}_{3}$ & 0.01 & 49.42 \\
\cline { 2 - 4 } & $\mathrm{CH}_{2} \mathrm{Cl}_{2}$ & 0.05 & 47.74 \\
\hline \multirow{6}{*}{$\mathrm{IV}$} & $\mathrm{CCl}_{4}$ & -0.28 & 61.78 \\
\cline { 2 - 4 } & $\mathrm{CHCl}_{3}$ & -0.17 & 57.31 \\
\cline { 2 - 4 } & $\mathrm{CH}_{2} \mathrm{Cl}_{2}$ & -0.07 & 53.13 \\
\hline
\end{tabular}

a) $\Delta \mathrm{G}_{\text {solvation }}$ calculated with PCM method at B3LYP/6-31G**//B3LYP/6$31 \mathrm{G}^{* *}$

b) $\Delta \mathrm{G}_{\text {solution }=}^{\mathrm{o}} \Delta \mathrm{G}_{\text {gas }}^{\mathrm{o}}+\Delta \Delta \mathrm{G}_{\text {solvation }}^{\mathrm{o}}$
From table 4, it can inferred that the axial and equatorial conformers are in equilibrium with a slightly larger concentration of the equatorial conformer in compound III, whereas in IV the axial conformer dominates. The equilibrium in compounds I and III are strongly equatorial in the gas phase and in solution of the solvents listed in table 4. The equatorial components in I and III shows the larger dipole moments and thereby their stabilization in solvents of higher dielectric constants can not be rule out and it is likely that the \% of axial conformers in compounds II and IV could become larger as $\varepsilon$ grows since their dipole moments is also larger (see fig.3)

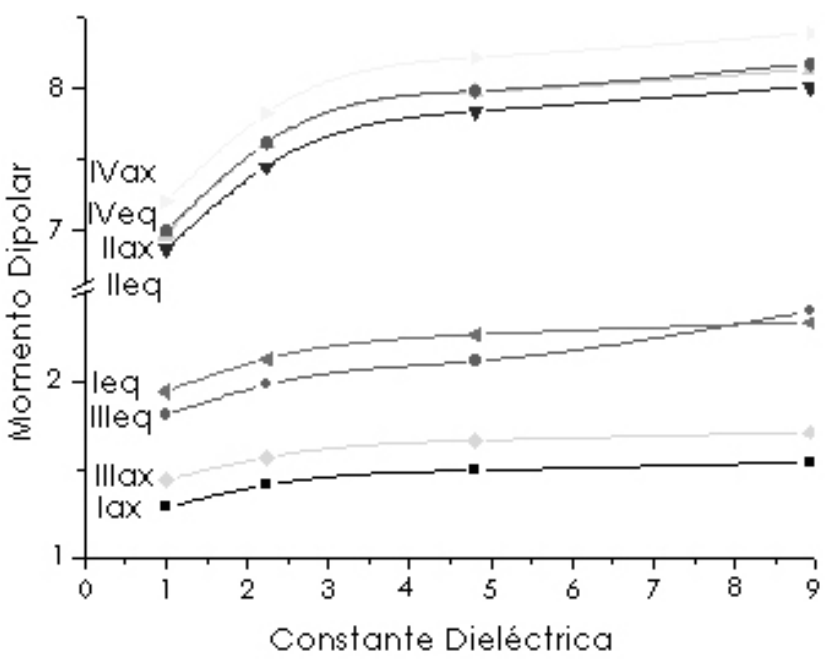

Fig. 3.- Dielectric constant and dipole moment relationship in all four compounds.

Reasoning out, the \% of the axial conformer in equilibrium II and IV Would also increase as the medium polarity increases. However, the dipole moments of the axial component of II and IV systems are larger than the equatorial one (see fig.3) and thereby such effect cannot be observed, most likely due to problems of PCM to handle the non-electrostatic contributions, such as cavitation and dispersion. It is also possible to think that large non-stabilizing interactions in the axial structures cannot be overcame for other dipole/dipole interactions with the solvent. In system II, the 1,3-interactions between $\mathrm{N}$-methyl group and the axial methyl on $\mathrm{C} 5$ would also play an important role. 


\section{TRANSITION STATES}

The transition states show , after NBO treatment at $\mathrm{B} 3 \mathrm{LYP} / 6-31 \mathrm{G}^{* *}$ level, nitrogen atom that reverses its equatorial character to an axial one through planar state as demonstrated by the increase of its "p" character in the lone pair of electrons (see table 5).

Table 5.- Atomic orbitals contributions to the hybrid orbital of the lone pair of electrons on nitrogen.

\begin{tabular}{|c|c|c|c|}
\hline & Ecuatorial & TS & Axial \\
\hline I & $15.54 \%$ s, $84.41 \% \mathrm{p}, 0.06 \% \mathrm{~d}$ & $0.01 \% \mathrm{~s}, 99.99 \% \mathrm{p}, 0.00 \% \mathrm{~d}$ & $10.96 \% \mathrm{~s}, 89.00 \% \mathrm{p}, 0.04 \% \mathrm{~d}$ \\
\hline II & $16.01 \% \mathrm{~s}, 83.93 \% \mathrm{p}, 0.05 \% \mathrm{~d}$ & $0.04 \% \mathrm{~s}, 99.96 \% \mathrm{p}, 0.00 \% \mathrm{~d}$ & $11.81 \% \mathrm{~s}, 88.14 \% \mathrm{p}, 0.04 \% \mathrm{~d}$ \\
\hline III & $12.79 \% \mathrm{~s}, 87.17 \% \mathrm{p}, 0.04 \% \mathrm{~d}$ & $0.01 \% \mathrm{~s}, 99.98 \% \mathrm{p}, 0.00 \% \mathrm{~d}$ & $14.04 \% \mathrm{~s}, 85.91 \% \mathrm{p}, 0.05 \% \mathrm{~d}$ \\
\hline IV & $16.18 \% \mathrm{~s}, 83.76 \% \mathrm{p}, 0.05 \% \mathrm{~d}$ & $0.06 \% \mathrm{~s}, 99.94 \% \mathrm{p}, 0.00 \% \mathrm{~d}$ & $14.40 \% \mathrm{~s}, 85.55 \% \mathrm{p}, 0.05 \% \mathrm{~d}$ \\
\hline
\end{tabular}

The TSs found exploring the potential energy surface describes well the inversion-rotation processes showing imaginary frequencies at $\mathrm{ca}^{180} \mathrm{~cm}^{-1}$. Two out of four TSs for the equatorial $₫$ axial interconversion were also characterized by following their reaction paths using IRC (intrinsic reaction coordinate) calculations. In both cases IRC (I and II) demonstrated that the corresponding TSs conduct to the reactants and products. The results of these calculations are shown in figure 4.

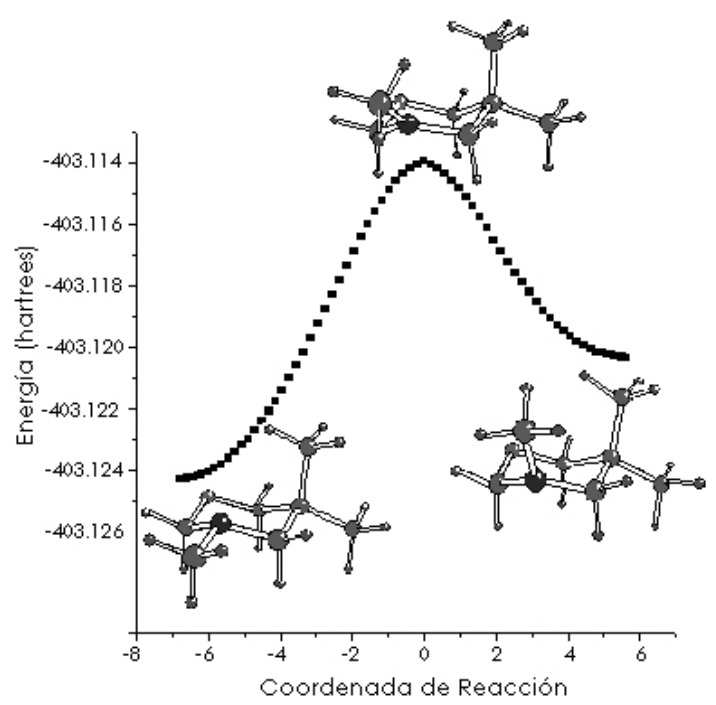

(a)

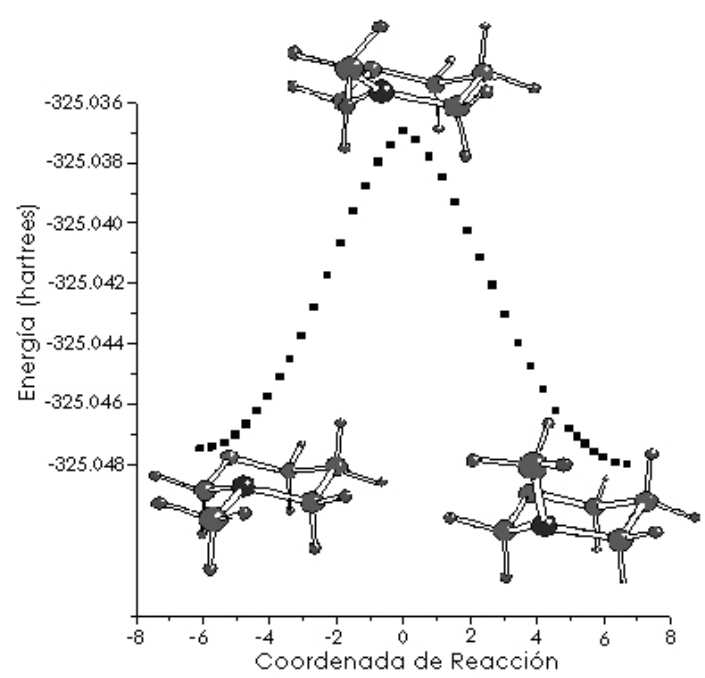

(b)

Figura 4.- IRC for (a) I and (b) III.. Plots obtained at HF/6-31G**.
The instability of TS for the equilibrium equatorial $₫$ axial in conformers I and II .indicates shows a strong repulsive effect between C5-CH3(ax) y N3$\mathrm{CH} 3$ subtituents. Similar to that observed at the axial minima I and II. In this sense a calculation of Brönstead coefficient yield a $\beta=0.564$ for the equilibrium in I and $\beta=0.547$ for II. In this way the structural similarities, imply that in both cases the corresponding TSs would show up the products alike. I and II cases follow well the postulate of Hammond and their endothermic character. For equilibrium III and IV Brönstead coefficients do not allow to infer if the TSs are reactant or product alike. In fact, values of $\beta$ of ca. 0.494 (III) and 0.490 (IV) indicate that neither the reactants nor products are good model for the TSs.

\section{CONCLUSIONS}

From the above results, some interesting conclusions can be outlined:

1.- The ring $\mathrm{O}$ and $\mathrm{N}$ sides dihedrals allow us to conclude that axial $\mathbf{I}$ and II conformers are more distorted at the $\mathrm{O}$ than at the $\mathrm{N}$-side.

2.- The presence of a large substituent on $\mathrm{C} 2$ exerts no much influence on the geometry of the oxazine ring. In fact, $\Delta \tau$ is just ca. $4^{\circ}$ for both axial and equatorial IV derivatives.

IIIax is an exception as no substituents on $\mathrm{C} 2$ or $\mathrm{C} 5$ are present and behaves like I and IIaxial conformers, though to a lesser extend

3.- Bulky p-nitrophenyl is a strong electron-withdrawing group and weakens and enlarges $\mathrm{C} 2 \mathrm{~N} 3$ distance, whereas methyl being an electronreleasing group counterbalances the above effect in conformer II. The O1C2 bond distances found in the series are longer for II and IV due to the pnitrophenyl group presence.

4.- Substituents on N3 in an equatorial position facilitates the chain/ring tautomerization in 1,3-oxazines. Electron-withdrawing groups increase the cyclic form contribution, whereas electron-releasing substituents would favor the chain forms.

5.- Comparison of the calculated and experimental values of chemical shifts and coupling constants indicates that the theoretical level used in the calculations is good enough to apply it in other cases to be studied.

6.- The instability of TS for the axial $\square$ equatorial I and II species suggests a strong repulsive effect between $\mathrm{C} 5-\mathrm{CH} 3(\mathrm{ax})$ y $\mathrm{N} 3-\mathrm{CH} 3$ subtituents. A Brönstead coefficient calculation yield a $\beta=0.564$ for the equilibrium in $\mathbf{I}$ and $\beta=0.547$ for II, implying that in both cases the corresponding TSs would look the products alike. I and II cases follow well Hammond postulate.

\section{ACKNOWLEDGEMENTS}

This work was partially supported by a grant $\left(\mathrm{N}^{\circ} 203,021.019-1.0\right)$ from the Universidad de Concepcion, Chile. S.M.H.O thanks CONICYT for a scholarship.

\section{REFERENCES}

1.- D. Gürne, T. J. Urbanski, J. Chem. Soc. 1912, (1959)

2.- W.Hofman, L. Stepaniak, T. J. Urbanski, M. J. Witanowski, J. Am. Chem Soc. 86, 554, (1964)

3.- J. M. Lehn, P. Linscheid, F. G. Riddell, Bull. Soc. Chim., France, 3, (1964) 1172, (1964) 
4.- F. G. Riddell, J. M. Lehn, J. Chem. Soc.(B) 1224, (1968).

5.- Y. Allingham, R. C. Cookson, T. A. Crabb, S. Vary, Tetrahedron 24, 4625 (1968).

6.- D. Gürne, T. J. Urbanski, L. Stepaniak, M. J. Witanowski, Tetrahedron Suplement 6, 211, (1963).

7.- A. R. Katrizky, V. J. Baker, F. M. S. Brito-Palma, J. Chem. Soc. (Perkin II) $1739,(1980)$.

8.- M. Tafazzoli, C. Suarez, N. S.Trae, C. B. LeMaster, C. L. LeMaster, J. Phys. Chem. 96, 10201, (1992).

9.- C. L. Perrin, J. D. Thoburn, S. Elsheimer, J, Org. Chem. 56, 7034, (1991).

10.- M. J. Frisch, G. W. Trucks, H. B. Schlegel, G. E. Scuseria, M. A. Robb, J. R. Cheeseman, J. A. Montgomery, Jr., T. Vreven, K. N. Kudin, J. C. Burant, J. M. Millam, S. S. Iyengar, J. Tomasi, V. Barone, B. Mennucci, M. Cossi, G. Scalmani, N. Rega, G. A. Petersson, H. Nakatsuji, M. Hada, M. Ehara, K. Toyota, R. Fukuda, J. Hasegawa, M. Ishida, T. Nakajima, Y. Honda, O. Kitao, H. Nakai, M. Klene, X. Li, J. E. Knox, H. P. Hratchian, J. B. Cross, V. Bakken, C. Adamo, J. Jaramillo, R. Gomperts, R. E. Stratmann, O. Yazyev, A. J. Austin, R. Cammi, C. Pomelli, J. W. Ochterski, P. Y. Ayala, K. Morokuma, G. A. Voth, P. Salvador, J. J. Dannenberg, V. G. Zakrzewski, S. Dapprich, A. D. Daniels, M. C. Strain, O. Farkas, D. K. Malick, A. D. Rabuck, K. Raghavachari, J. B. Foresman, J. V. Ortiz, Q. Cui, A. G. Baboul, S. Clifford, J. Cioslowski, B. B. Stefanov, G. Liu, A. Liashenko, P. Piskorz, I. Komaromi, R. L. Martin, D. J. Fox, T. Keith, M. A. Al-Laham, C. Y. Peng, A. Nanayakkara, M. Challacombe, P. M. W. Gill, B. Johnson, W. Chen, M. W. Wong, C. Gonzalez and J. A. Pople. Gaussian 03, Revision D.01,Gaussian, Inc., Wallingford CT, 2004.

11.- J. G. Contreras, S. M. Hurtado, M. M. C. Ferreira, L. Gerli and R. Castillo.J. Chil. Chem. Soc. 50, 731, (2005).
12.- K. Wolisky, J. F. Hilton, P. Pulay, J. Am. Chem. Soc., 112, 8251, (1990) 13.- J. R. Cheeseman, G. W. Trucks, T. A. Keith, M . J. Frisch, J. Chem. Phys., 104, 5497, (1996).

14.- J. G. Contreras, L. A. Gerli, J. Chil. Chem, Soc., 52, 1271, (2007)

15.- A. E. Reed, R. B. Weinstock, F. Weinhold, J. Chem. Phys., 83, 735 (1985).

16.- A. E. Reed, L. A. Curtis, F. Weinhold, Chem. Rev. 88, 899, (1988).

17.- I. V. Alabugin, T.A. Zeidan, J. Am. Chem. Soc., 124, 3175, (2002).

18.- (a)Cope, A. C.; Hancock, E. M. J. Am. Chem. Soc. 64, 1503, (1966), (b)Bergmann E.D., Gil-Av E., Pinchas S., J. Am. Chem. Soc. 75, 358, (1953) (c) Fülöp F., Pihlaja K., Mattinen J., Bernáth G., J. Org. Chem. 52, 3821, (1987) (d) Fülöp F., Pihlaja K., Mattinen J., Neuvonen K., Bernáth G., Argay G., Calman A., J. Org. Chem. 58, 1967, (1993) (e) Neuvonen K., Fülöp F., Neuvonen H., Koch A., Kleinpeter E., Pihlaja K., J. Org. Chem. 66, 4132, (2001) (f) Szatmári I., Martinek T. A., Lázár L., Koch A., Kleinpeter E., Neuvonen K., Fülöp F., J. Org. Chem. 69, 3645, (2004).

19.- (a)Belgodere E., Bossio R., Parrini V., Pepino R., J. Heterocycl. Chem. 17, 1629 (1980), (b) Sabed A. A. H., Ebraheem E.K. Can. J. Spectrosc. 28169), (1983).

20.- (a) G.S. Hammond., J. Am. Chem. Soc. 77, 334, (1955) (b) R. A. Marcus., Annu. Rev. Phys. Chem. 15, 155, (1964).

21.- (a) S. Miertus, E. Scrocco and J. Tomasi., J. Chem. Phys. 55, 117, (1981) (b)J. Tomasi, R. Bonaccorsi, R. Cammi and F.J. Olivares del Valle., J. Molec. Struct. Theochem. 234, 401, (1991).

22.- M. Hurtado, J. G.Contreras, A. Matamala, O. Mo, M. Yañez. New J. Chem. 32, 2209 (2008). 